\title{
SOME EXAMPLES OF TAUBERIAN OPERATORS
}

\author{
TERESA ALVAREZ AND MANUEL GONZALEZ
}

(Communicated by Palle E. T. Jorgensen)

\begin{abstract}
We study some examples of tauberian operators and show that the second conjugate of a tauberian operator is not always tauberian, answering a question of Kalton and Wilansky. Also we show that the class of tauberian operators is not open, although tauberian operators in the boundary of the class must have nonclosed range.
\end{abstract}

\section{INTRODUCTION}

Tauberian operators appeared in a problem of summability [4] and were studied by Kalton and Wilansky [10] and other authors [1, 5, 7, 11, 12]. Recently they have received some attention because they form a broader class than that of isomorphisms (into), but yet they preserve some isomorphic properties of Banach spaces $[8,9]$.

Let $T^{*} \in L\left(Y^{*}, X^{*}\right)$ and $J(X)$ denote the conjugate operator of a continuous linear operator $T \in L(X, Y)$ and the immersion of a Banach space $X$ into the second dual $X^{* *}$.

$T$ is tauberian when $T^{* *-1} J(Y)=J(X)$.

Upper semi-Fredholm operators (operators with closed range and finitedimensional kernel) are trivial examples of tauberian operators, since they are isomorphisms up to finite-dimensional subspace.

The main source of nontrivial examples of tauberian operators is the celebrated factorization of Davis et al. (DFJP factorization) [3]. However, these operators are still a very special class of tauberian operators.

In this paper, we show that all the conjugates of even order of the second factor in the DFJP factorization are tauberian operators. Then, using a construction of Bellenot [2], we obtain a Banach space and a tauberian operator $T$ in this space such that the second conjugate $T^{* *}$ is not tauberian, answering a question of Kalton and Wilansky [10]. Finally, we present a simple example showing that the class of tauberian operators is not always open (another

Received by the editors August 17, 1989 and, in revised form, March 5, 1990.

1980 Mathematics Subject Classification (1985 Revision). Primary 47B05.

Key words and phrases. Tauberian operator.

Supported in part by DGICYT grant PB88-0417. 
such example can be found in [11]), and we prove that tauberian operators with closed range do not belong to the boundary of this class.

\section{TAUBERIAN OPERATORS IN THE DFJP FACTORIZATION}

Let \|\|$_{n}(n=1,2, \ldots)$ be equivalent norms in a Banach space $X$. We define $X_{n}:=\left(X,\|\|_{n}\right)$,

$$
l_{2}\left(X_{n}\right):=\left\{\left(x_{n}\right): x_{n} \in X_{n} \text { and } \sum\left\|x_{n}\right\|^{2}<\infty\right\}
$$

and

$$
D:=\left\{\left(x_{n}\right) \in l_{2}\left(X_{n}\right): x_{n}=x_{1} \text { for every } n\right\} .
$$

It is clear that $l_{2}\left(X_{n}\right)$ is a Banach space and $D$ is a closed subspace of $l_{2}\left(X_{n}\right)$. We shall denote by $i$ the inclusion of $D$ into $l_{2}\left(X_{n}\right)$; by $P$, the projection of $l_{2}\left(X_{n}\right)$ onto $X_{1}$; by $j, P i$; and by $T^{(n)}$, the $n$th conjugate of an operator $T \in L(X, Y): T^{(0)}=T, T^{(1)}=T^{*}, T^{(2)}=T^{* *}, \ldots$. The second factor in the DFJP factorization is a particular case of $j$.

Proposition 1. $j^{(2 n)}$ is a tauberian operator for every $n=0,1,2, \ldots$.

Proof. Note that the space $D^{* *}$ can be identified with the second annihilator $D^{00}=\left\{\left(F_{n}\right) \in l_{2}\left(X_{n}^{* *}\right): F_{n}=F_{1}\right.$ for every $\left.n\right\}$, and $l_{2}\left(X_{n}\right)^{* *}=l_{2}\left(X_{n}^{* *}\right)$. Then, for every $(F, F, F, \ldots) \in D^{* *}$, we have $j^{* *}(F, F, \ldots)=P^{* *}(F, F, \ldots)=$ $F$, and it is clear that $j$ is tauberian. The same argument is valid for the remaining conjugates of even order of $j$.

\section{TAUBERIAN OPERATORS WITH NONTAUBERIAN SECOND CONJUGATE}

For every operator $T \in L(X, Y)$, we can consider a canonically associated operator $T_{q} \in L\left(X^{* *} / X, Y^{* *} / Y\right)$ given by

$$
T_{q}(F+X)=T^{* *} F+Y \quad\left(F \in X^{* *}\right) .
$$

This associated operator allows us to characterize tauberian operators in the following way.

Proposition $2[12 ; 6, \S 2.8] . T$ is tauberian if and only if $T_{q}$ is injective.

Moreover, we can identify $\left(T_{q}\right)^{* *}=\left(T^{* *}\right)_{q}$.

Observation 3. From the above characterization it is clear that given Banach spaces $X, Y$ with $X^{* *} / X$ reflexive, we have that $T \in L(X, Y)$ is tauberian if and only if $T^{* *}$ is tauberian.

Now we shall present an example of a tauberian operator $S$ such that $S^{* *}$ is not tauberian. In order to do that, we shall construct an injective operator $T \in L\left(l_{1}\right)$ such that $T^{* *}$ is not injective, and a Banach space $Z$ and $S \in L(Z)$ in such a form that $Z^{* *} / Z \equiv l_{1}$ and $S_{q} \equiv T$.

We use a particular case of a construction of Bellenot [2] giving a Banach space $Z$ such that $Z^{* *} / Z$ is isometric to $l_{1}$. 
We denote by $e_{n}$ the unit vector basis of $l_{1}$, by $X_{n}$ the subspace of $l_{1}$ generated by $\left\{e_{1}, \ldots, e_{n}\right\}$, and by $P_{n}$ the projection from $l_{1}$ onto $X_{n}$, and we let

where

$$
J\left(X_{n}\right):=\left\{\left(x_{n}\right): x_{n} \in X_{n},\left\|x_{n}\right\|_{1} \rightarrow 0 \text { and }\left\|\left(x_{n}\right)\right\|_{J}<\infty\right\},
$$

\section{Theorem 4}

$$
2\left\|\left(x_{n}\right)\right\|_{J}^{2}:=\sup \left\{\left(\sum_{i=1}^{k-1}\left\|x_{n_{i+1}}-x_{n_{i}}\right\|^{2}\right)+\left\|x_{n_{k}}\right\|^{2}: n_{1}<n_{2}<\cdots<n_{k}\right\} .
$$

(a) $\left(J\left(X_{n}\right),\|\|_{J}\right)$ is a Banach space.

(b) $J\left(X_{n}\right)^{* *}=\left\{\left(x_{n}\right): x_{n} \in X_{n},\left\|\left(x_{n}\right)\right\|_{J}<\infty\right\}$.

(c) If $\left(x_{n}\right) \in J\left(X_{n}\right)^{* *}$, then $\left\{x_{n}\right\}$ is convergent in $l_{1}$.

(d) $J\left(X_{n}\right)^{* *} / J\left(X_{n}\right)$ is isometric to $l_{1}$.

Proof. (a) It is standard.

(b) It is enough to note that $\left\{X_{n}\right\}$ is a shrinking decomposition of $J\left(X_{n}\right)$ $[2, \S 1.1]$.

(c) Suppose $\left\{x_{n}\right\}$ is not convergent in $l_{1}$. Then there exists $\delta>0$ and $n_{1}<m_{1}<n_{2}<m_{2}<\cdots$ such that $\left\|x_{m_{i}}-x_{n_{i}}\right\|>\delta$. Then we would have $\left\|\left(x_{n}\right)\right\|_{J}=\infty$, a contradiction.

(d) It is enough to verify that the map

$$
U:\left(x_{n}\right)+J\left(X_{n}\right) \in J\left(X_{n}\right)^{* *} / J\left(X_{n}\right) \rightarrow \lim x_{n} \in l_{1}
$$

is an isometry. We have left the details for the reader.

Now consider the operator $T \in L\left(l_{1}\right)$, given by $T\left(x_{n}\right):=\left(x_{n} / n\right)$. It is easy to see that $T$ is a compact operator. Then $T^{*}$ does not have dense range, since $l_{\infty}$ is nonseparable; hence $T^{* *}$ is not injective. Note that $T$ leaves invariant the subspaces $X_{n}$ of $l_{1}$ considered above, and it commutes with the projections $P_{n}$.

On the other hand, let us consider the operator $S$, defined in $J\left(X_{n}\right)$ by

\section{Proposition 5}

$$
S\left(x_{n}\right)=\left(T x_{n}\right), \quad x_{n} \in X_{n} .
$$

(a) $S \in L\left(J\left(X_{n}\right)\right)$.

(b) $S_{q} \equiv T$ (up to an isometry).

(c) $S$ is tauberian, but $S^{* *}$ is not tauberian.

Proof. (a) From $\left\|T x_{n_{i+1}}-T x_{n_{i}}\right\| \leq\|T\|\left\|x_{n_{i+1}}-x_{n_{i}}\right\|$ and the definition of \|\|$_{J}$, it follows easily that $\|S\| \leq\|T\|$.

(b) Take $\left(x_{n}\right) \in J\left(X_{n}\right)^{* *}$, and let $U$ be the isometry in the proof of Theorem 4: $U\left(\left(x_{n}\right)+J\left(X_{n}\right)\right)=\lim x_{n}$. We define $u:=\lim x_{n}$.

Note that $\left(P_{n} u\right)+J\left(X_{n}\right)=\left(x_{n}\right)+J\left(X_{n}\right)$, since both sequences have the same limit. Then we have $T U\left(\left(x_{n}\right)+J\left(X_{n}\right)\right)=T u=U\left(\left(P_{n} T u\right)+J\left(X_{n}\right)\right)=$ $U\left(S\left(P_{n} u\right)+J\left(X_{n}\right)\right)=U S_{q}\left(\left(P_{n} u\right)+J\left(X_{n}\right)\right)=U S_{q}\left(\left(x_{n}\right)+J\left(X_{n}\right)\right)$.

(c) This is a consequence of (b) and the properties of $T$. 
Observation 6. Denote by WCo the class of all weakly compact operators. The following characterization of tauberian operators was obtained in [7]: An operator $T \in L(X, Y)$ is tauberian if and only if, for every space $Z$ and $A \in$ $L(Z, X), T A \in \mathrm{WCo} \Rightarrow A \in \mathrm{WCo}$.

We can consider a dual definition: $T \in L(X, Y)$ is cotauberian if and only if, for every space $Z$ and $B \in L(Y, Z), B T \in \mathrm{WCo} \Rightarrow B \in \mathrm{WCo}$.

We obtain that $T \in L(X, Y)$ is cotauberian if and only if $T^{*}$ is tauberian [6], [7]. In fact, cotauberian operators were introduced in [6] (see also [11]) as operators whose conjugates are tauberian, and the above definition is a characterization in [7]. Moreover, it is easy to see that $T^{*}$ cotauberian implies $T$ tauberian. However, the above example shows that the remaining implication is not true.

\section{OPERATORS IN THE BOUNDARY OF THE SET OF TAUBERIAN OPERATORS}

In general, the class of tauberian operators is not open in $L(X, Y)$. This fact has been observed by Tacon [11]. We now present a very easy example showing it.

Let $X$ be a nonreflexive Banach space, and consider the operator defined by

$$
T:\left(x_{k}\right) \in l_{2}(X) \rightarrow\left(k^{-1} x_{k}\right) \in l_{2}(X) .
$$

Since $l_{2}(X)^{* *}$ can be identified with $l_{2}\left(X^{* *}\right)$, and $T^{* *}$ acts in the same form as $T$, it is clear that $T$ and all its conjugates of even order are tauberian. However, we can approximate $T$ by the operators $T_{n}$ given by

$$
T_{n}:\left(x_{k}\right) \in l_{2}(X) \rightarrow\left(x_{1}, 2^{-1} x_{2}, \ldots, n^{-1} x_{n}, 0,0, \ldots\right) \in l_{2}(X) .
$$

In fact we have $\left\|T_{n}-T\right\|=1 / n$, but $T_{n}$ is not tauberian since its kernel is not reflexive.

In some situations we can assure that the class of tauberian operators has a nonempty interior.

Proposition 7. Tauberian operators with closed range do not belong to the boundary of the class of tauberian operators.

Proof. If $T \in L(X, Y)$ is tauberian with closed range, then the associated operator $T_{q} \in L\left(X^{* *} / X, Y^{* *} / Y\right)$ is injective with closed range [12, §2.6]. Moreover, $\left\|(T-S)_{q}\right\| \leq\|T-S\|[12, \S 2.3]$. Hence the result follows from the openness of the class of injective operators with closed range.

\section{ACKNOWLEDGMENT}

The second author is grateful to S. Bellenot for a useful conversation.

\section{REFERENCES}

1. K. Astala and H.-O. Tylli, Seminorms related to weak compactness and to tauberian operators, Math. Proc. Cambridge Phil. Soc. 107 (1990), 367-375.

2. S. Bellenot, The J-sum of Banach spaces, J. Funct. Anal. 48 (1982), 95-106. 
3. W. Davis, T. Figiel, W. Johnson, and A. Pelczynski, Factoring weakly compact operators, J. Funct. Anal. 17 (1974), 311-327.

4. D. J. H. Garling and A. Wilansky, On a summability theorem of Berg, Crawford and Whitley, Proc. Cambridge Phil. Soc. 71 (1972), 495-497.

5. M. Gonzalez and V. M. Onieva, Semi-Fredholm operators and semigroups associated with some classical operator ideals, Proc. Roy. Irish Acad. 88A (1988), 35-38.

6. _ Semi-Fredholm operators and semigroups associated with some classical operator ideals-II, Proc. Roy. Irish Acad. 88A (1988), 119-124.

7. _ Characterizations of tauberian operators and other semigroups of operators, Proc. Amer. Math. Soc. 108 (1990), 399-405.

8. R. Neidinger, Properties of tauberian operators on Banach spaces, Ph.D. thesis, Univ. Texas, 1984.

9. R. Neidinger and H. Rosenthal, Norm-attainment on linear functionals on subspaces and characterizations of tauberian operators, Pacific J. Math. 118 (1985), 215-228.

10. N. Kalton and A. Wilansky, Tauberian operators in Banach spaces, Proc. Amer. Math. Soc. 57 (1976), 251-255.

11 D. G. Tacon, Generalized semi-Fredholm transformations, J. Austral. Math. Soc. A34 (1983), 60-70.

12. K. W. Yang, The generalized Fredholm operators, Trans. Amer. Math. Soc. 219 (1976), 313-326.

Departamento de Matemáticas, Facultad de Ciencias, Universidad de Oviedo, 33004 Oviedo, SPAIN

Departamento de Matemáticas, Facultad de Ciencias, Universidad de Cantabria, 39005 SANTANDER, SPAIN 